\title{
Advancing Critical Care in the ICU: A Human-Centered Biomedical Data Visualization Systems
}

\author{
Anthony Faiola and Chris Newlon \\ Indiana University - School of Informatics, \\ 535 W. Michigan Street, Indianapolis, IN 46202, USA \\ \{faiola, cnewlon $\}$ @iupui.edu
}

\begin{abstract}
The purpose of this research is to provide medical clinicians with a new technology for interpreting large and diverse datasets to expedite critical care decision-making in the ICU. We refer to this technology as the medical information visualization assistant (MIVA). MIVA delivers multivariate biometric (bedside) data via a visualization display by transforming and organizing it into temporal resolutions that can provide contextual knowledge to clinicians. The result is a spatial organization of multiple datasets that allows rapid analysis and interpretation of trends. Findings from the usability study of the MIVA static prototype and heuristic inspection of the dynamic prototype suggest that using MIVA can yield faster and more accurate results. Furthermore, comments from the majority of the experimental group and the heuristic inspectors indicate that MIVA can facilitate clinical task flow in context-dependent health care settings.
\end{abstract}

Keywords: Biomedical data visualization, human-computer interaction, health care, health information technology, interface design.

\section{Introduction}

Healthcare is a risky business. Patients are at risk from disease and poor health habits, with interventions like medicine and surgery carrying the risk of adverse effects. The Institute of Medicine's report To Err is Human: Building a Safer Health System [1] highlighted the risk of adverse medical events in clinical settings, with patient deaths in U.S. hospitals annually exceeding 44,000 , at a cost of $\$ 38$ billion. In extreme instances lives have been lost due to poor software design. For example, between 1985 and 1987 the problematic design of the Therac- 25 radiation system resulted in massive overdoses of radiation and at least five patient deaths [2]. An investigation into user error pointed at the interface design, which had a complex and misleading operator console. Ultimately, a lack of humancentered design and testing techniques resulted in poor design and a range of critical errors in the interaction routines.

The technologies and information systems needed by clinicians can produce adverse effects when they do not yield practical solutions during implementation. For example, systems that display large amounts of complex patient data can result in confusion when designed without knowledge of human-computer interaction (HCI) and usability principles. This body of knowledge includes human factors engineering, as well as a social science approach to human-centered design. Human-centered design employs cognitive

M.M. Robertson (Ed.): Ergonomics and Health Aspects, HCII 2011, LNCS 6779, pp. 119-128, 2011. Springer-Verlag Berlin Heidelberg 2011

Faiola, A., \& Newlon, C. (2011). Advancing critical care in the ICU: a human-centered biomedical data visualization systems. In Ergonomics and Health Aspects of Work with Computers (pp. 119-128). Springer Berlin Heidelberg. http://dx.doi.org/10.1007/978-3-642-21716-6_13 
psychology, anthropology, and sociology, as well as interface and interaction design, which take into consideration the specific medical device, the users, the development process, and the organization and context of use. Human-centered design in health information technology (HIT) can improve clinical care. For instance, it can address preventative medicine compliance issues in unusually complex health care environments, specifically with respect to the number of interactions between clinicians and data visualization systems [3]. Designing and implementing HIT is not about IT per se nor an isolated use of traditional usability or human factors engineering. Rather, as Zhang [4] argues, HIT design is a "human project about human-centered computing such as usability, workflow, organizational change, medical error, and process reengineering" ( $p$. 1). In the past, too much of $\mathrm{HCI}$ and usability engineering has been a superficial enterprise of designing form (e.g., the graphic user interface) rather than context-centered interaction based on an in-depth analysis of cognition, task, symbol, and workflow. It is no wonder that HIT often hinders health care. Bernstam et al [3] point out that "there is evidence of significant, unintended and deleterious effects of well-meaning HIT efforts" (p. 1).

The intensive care unit (ICU) is a uniquely complex environment for which computing technology needs to be developed. The authors call for HIT based on novel design principles and practices. ICU clinical decisions are sometimes made with data from multiple sources, changing circumstances, and limited clinician knowledge. Such situations dictate quick adjustments in the planning of actions. In many cases, clinical error is the result of cognitive overload due to the inability of humans to adapt to excessive and complex physical, social, and cultural environments [5]. Horsky, Zhang, and Patel [5] note that a "lack of attention to the principles of human-computer interaction (HCI) in clinical software design is becoming a critical safety hazard" (p. 264). The authors concur that there is a need for a framework of human-centered design principles that are grounded in HCI theory specifically developed for clinical environments. Unfortunately, a health care culture still remains that accepts poorly designed systems. As Johnson, Johnson, and Zhang [6] and Horsky, Zhang, and Patel [5] all argue, clinicians should not tolerate such conditions. Rather, they should demand that health care technologies be designed to match user capabilities [6].

The ICU is a setting where there can be an enormous flow of data. Patients can have significant organ system derangement, as well as limited physiological reserve. Therefore, optimal medical care requires close and concentrated monitoring of organ function, frequent multimodal diagnostic testing, and many consultations from subspecialty physicians. In this type of intensive critical care space, physicians may find it difficult to make rapid and accurate evaluations of a patient's condition. This is because there is an overwhelming volume of data generated from multiple sources on a moment-by-moment basis. Data streaming from medical devices include continuous automatic physiological monitoring and intermittently determined data that is gathered from various diagnostic testing devices. These are combined with traditional bedside paper reports. In addition to patient-generated data, there are vast arrays of clinical data generated that document the patient's treatments, including drug, respiratory, and physical therapy, surgical procedures, and many other clinical interventions [7].

Clinical decisions pertaining to the care of patients are best made when physicians can easily organize and understand the vast flood of data from these various sources. Unfortunately, physicians and other critical care providers have to retrieve this data from various locations and organize it into a cohesive profile of the patient's current condition. Furthermore, the data that is retrieved from these diverse sources is usually presented in a 
form that does not allow trends and relationships between co-variables to be immediately recognized. As a result, the process of monitoring, evaluating and treating the critically ill is labor intensive, time-consuming, and demands that clinicians analyze data in text and numeric form. These difficulties are compounded by the highly stressful, time-restricted character of the ICU environment.

Although the monitoring devices of a modern ICU are intended to support the immediate recognition of problems, only a limited amount of historical data can be reviewed on the monitor on-the-fly. For example, although current monitoring devices maintain a reasonably comprehensive log of a patient's critical care period, its output is in conventional spreadsheet form, with, at best, a few parameters displayed as $\mathrm{X}-\mathrm{Y}$ plots. Some existing systems provide some degree of relational multi-source review of data, but have interfaces that are often antiquated, resembling a graphic user interface (GUI) from an early 1990s version of Microsoft Windows. As a result, clinician's find it difficult and cumbersome to make rapid critical evaluations of a patient's condition.

What is needed is an integrated platform through which data can be organized into a uniform, multivariate data visualization as the primary means to represent and manage complex patient critical care data. This includes intuitive human-centered applications of data that allow access to various time resolutions and subsets of patient data over minutes, hours, and days. This system must allow clinicians to control and compare current and past events and trends to better explore and examine longitudinal relationships in patient data.

\section{Previous Medical Information Visualization Systems}

One of the earliest designers to address medical data visualization was Edward Tufte [8]. Although his work was not multivariate, his attempt at consolidating, integrating, and visualizing a broad array of data was a first step in addressing this need in the health care area. Tufte [8] effectively combined several timescales in one horizontal axis. Subsequent data visualization systems for the ICU have attempted to connect time-oriented information to a coherent interactive visualization, presenting different interactive visualization techniques and enabling the users to reveal the data at several levels of detail and abstraction [9]. The first known data visualization system for the ICU was developed by Horn, Popow, and Unterasinger [7]. Their computer-based patient data management system (PDMS) stored data that had been collected online or entered manually within a specified time frame. The purpose of this system was to quickly generate and observe graphics for detecting changing patient parameters. Horn et al [7] described PDMS as a data visualizing, time-oriented system of analysis of electronic patient records in an ICU. The focus of the product was to make it easier for clinicians to quickly assess the overall situation of a patient and to recognize essential changes over time. They described the method of the system as using graphics to sketch the most relevant parameters for characterizing an ICU patient's situation over a 24 hour time frame presented in one display.

The authors argue that such medical devices and HIT system applications must be human-centered in their initial and iterative design implementation to ensure that such systems can adequately and safely support clinical care. A medical data visualization system is proposed, with several initial pilot studies that suggest that it can support clinical work in the ICU or other data rich health care environments. 


\section{Proposed System}

The authors recently designed and developed a medical data visualization system, referred to as the Medical Information Visualization Assistant (MIVA). The purpose of MIVA is to provide valuable assistance for data analysis and decision making in a real-world critical care environment. In particular, MIVA provides a means to integrate and display data from a range of bedside biometric devices and health care provider data sources in the ICU. According to Bellazzi and Zupan [10], the decision-making process should always be supported by relevant and reliable data that is presented in a "context-related" fashion. MIVA attempts to do this by contextualizing data in visualization-form to better respond to an immediate patient status. Also, data visualizations can be prioritized according to particular clinical problems, while being customizable on-the-fly.

For example, an anesthesiologist will have different data visualization priorities than a cardiologist. Similarly, a child who has undergone cardiac surgery may have different data presentation requirements than a 70-year-old man who has an acute exacerbation of chronic respiratory disease. Thus, data visualization must be tailored to the patient, the disease, the consulting specialty, and/or the acute medical problem at the moment or historically. MIVA provides a uniquely new solution for addressing contextual health care issues with a multivariate data visualization.

MIVA improves diagnosis speed and accuracy by recognizing essential changes to multiple and relational physiological data over a designated time frame. Using selection menus, physicians control the necessary data sources, time periods, and time resolutions to narrow down their diagnosis and final assessment of a patient's condition. Hence, the MIVA interface has been designed to maximize the clinician's ability to control what data is visualized during a specific context-related patient episode or general periodic diagnosis.

\section{Design Process}

Stage 1. Early discussions centered around possible design models for visualizing data using Tufte's [8] visualization research and publications. After several visits to existing bedside and visualization systems in the Intensive Care Unit, a clearer understanding of the complexity of data delivery to health care staff was realized. The first visualization sketches focused on five data biometrics: iNO (nitric oxide), RR (ventilator rate), PAP (pulmonary artery pressure), $\mathrm{ABP}$ (arterial pressure), and $\mathrm{SvO}_{2}$ (venous oxygen saturation). See Figure 2-A. This sketch evolved into a series of visualization design iterations. See Figure 2-B through 2-D. This phase of the design process included ongoing informal focus groups with medical faculty about placement of data points, system of time display (minutes, hours, days), size of numeric information, and general location of biomedical, time, and numeric information. Most critical was the need of clinicians to see data visualized in various histories, with various levels of time and data resolution.

Stage 2. The next stage of visualization design involved HCI graduate students assisting in concept development of the interface and visualization tools. Students were instructed to use the authors' preliminary work (shown in Figure 2, A-D) as the basis for their continued design. This design phase included ethnographic studies at the ICU at Riley Hospital, including photographs of the ICU environment and of bedside devices. The 
concept of a "scrubber" was borrowed from audio editing software, as seen in the program ProTools. In the case of longitudinal patient data, the scrubber tool would allow the user to identify a specific point in time and instantly obtain readings for all intersecting points between the $\mathrm{Y}$ axis and the scrubber sitting at the $\mathrm{X}$ axis (or point in time). See Figure 3. This stage also yielded a published paper discussing its development [11].

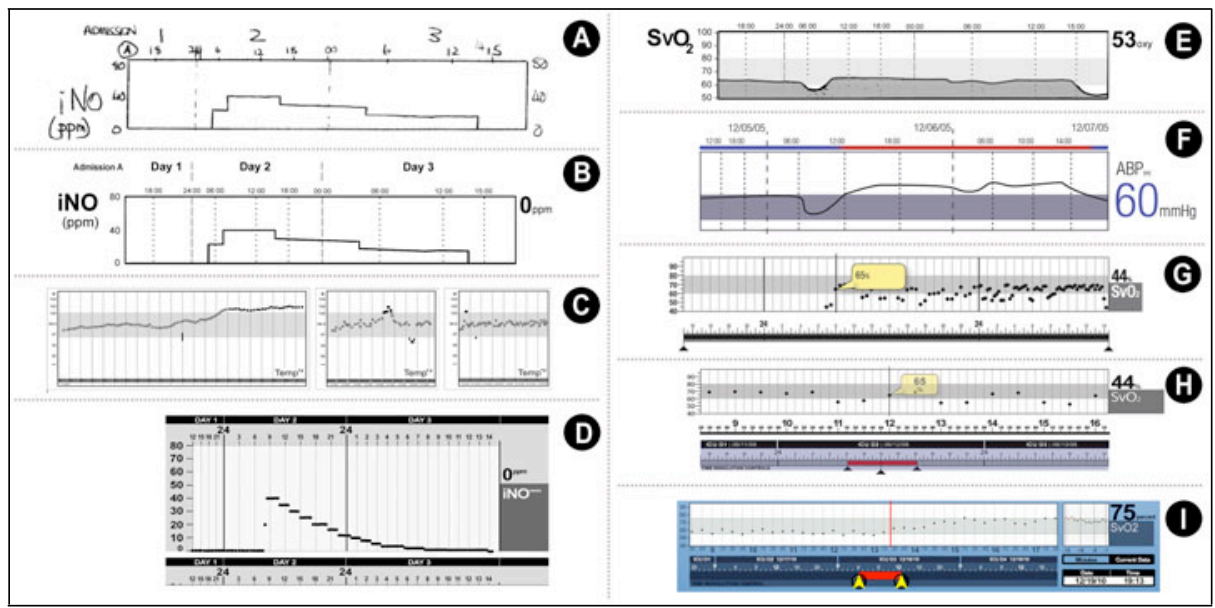

Fig. 2. A-I. Illustration of development of MIVA interface over a four year period

The outcome of our joint efforts was the creation of several static prototypes, with recommendations and solutions for technically integrating the data from multiple sources. As a result, the completed designs shown in Figure 2, E$\mathrm{G}$, suggested a further development of the MIVA interface. In this second stage of the design process, the team worked to resolve several major design issues related to clinician workflow and cognitive modeling.

Stage 3. The primary mission of this stage was to further enhance the existing interface and

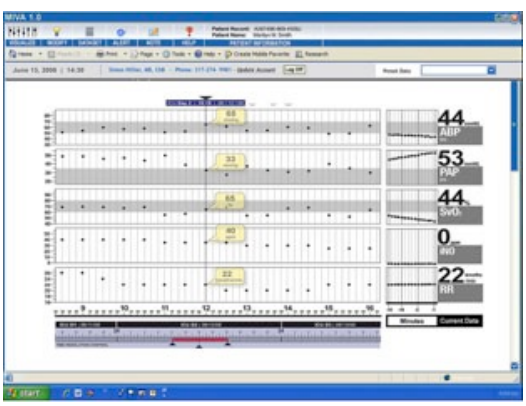

Fig. 3. One example of the static MIVA interface used in first usability study prepare detailed static prototypes for the first usability study. Figure 2, H, shows the further development of the visualization line of a single biometric parameter, as compared to the forgoing designs. Figure 3 shows the full design that was used in the first usability study, to be discussed below. Finally, Figure 2, I, shows the final and current state of the interface after the findings of the first usability study.

Stage 4. This stage included the creation of a dynamic prototype using Flash Action Script. See Figure 4 for the current state of the visualization interface. As noted above, MIVA's multivariate, relational display of information allows clinicians to observe contextual changes in patient data over selected periods of time at various time resolutions. A 
multivariate comparative analysis is vital if physicians want to do a relational diagnosis. 'Also, the longitudinal data streams from each parameter have time-aligned clinical and intervention notes that are inserted at the appropriate point in time with icons above the parameter visualization display. See Figure 5. Figure 4 shows the Display Sets from which clinicians can choose a range of physiological parameters. The clinician must first select and drag-n-drop the needed parameter into the primary visualization display (e.g., $\mathrm{Ca}$, iNO, or $\mathrm{CO} 2$ ). Figure 5 also shows the drag-n-drop model used to change physiological parameters.

The physiological parameter (LAP) is clicked (Figure 4, A) and dragged onto the visualization platform to the right (Figure 4, B), and the new parameter with its corresponding data is now in place on the visualization platform (Figure 4, C). The data streams from bedside monitoring devices reach the MIVA interface after being routed through the hospital's electronic medical record (EMR) system. The clinical and intervention notes entered by the

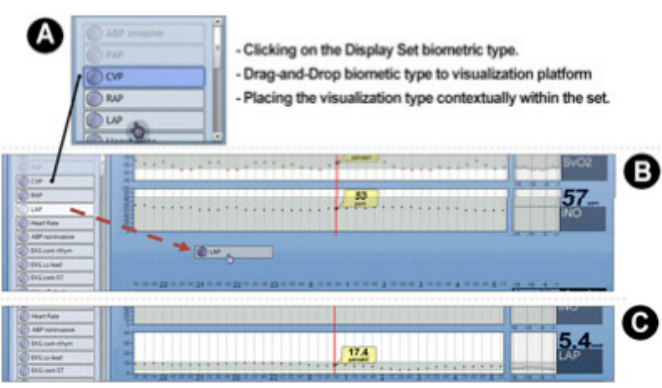

Fig. 4. A-C. Illustrates the drag-n-drop process for inputting a new parameter into the visualization display health care practitioners will also be sent to the EMR, in addition to being displayed on the timeline. In this way, MIVA becomes another node integrated into the hospital's network.

\section{Human-Centered Design and Testing of the MIVA Interface}

\subsection{MIVA Study One - Static Prototype}

A usability and clinical test was performed on the MIVA Phase One prototype to add to the value of this project. The MIVA study used control and experimental groups $(n 16)$ from the medical resident population at Indiana University School of Medicine to compare traditional methods of gathering and assessing critical patient data using paper charts (Figure 6) versus MIVA (Figure 3).

- Participants: The study used participants from the medical resident population at Indiana University School of Medicine, Indianapolis, IN. The control group and the experimental group consisted of eight participants each, $n 16$. The average experience level of the resident participants across the control group was 3.38 years, of which seven out of eight were male. The average experience level of the resident participants across the experimental group was 3.25 years, of which six of eight were male.

- Methods: The study was conducted in the Indiana University Schools of Medicine and Nursing. Participants from each group were given the same clinical scenario and eight questions to answer about the scenario. As each participant read the question, he or she sought the answer from either the traditional medical paper charts or MIVA. Participants in the control group answered the questions using traditional paper-based charts as their data source, and participants from the experimental group used screen shots of the MIVA interface to answer the same questions. The screen captured interface images were not interactive, but were prepared and displayed sequentially in 
a PowerPoint presentation, shown in Figure 3. Experimental group participants were directed to walk through the slide presentation, one interface slide at a time, to ascertain the correct answer to the eight questions. Both the control and experimental group participants were provided a 3-5 minute priming session (before the test session) to understand the basic placement of data on the medical charts and MIVA interface. After completion of the eight tasks, participants from both groups answered a post-test questionnaire.

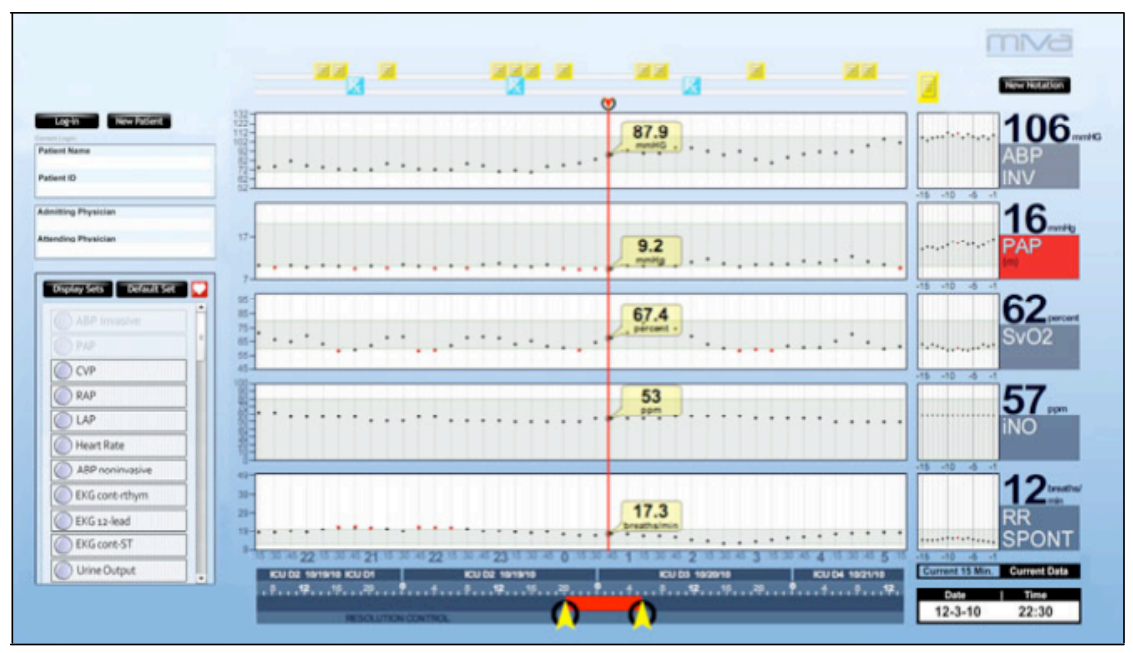

Fig. 5. Example of MIVA interface with control panel and data visualizations. Note the clinical and intervention notes entered by clinicians at top, designated with icons above the visualization display.

- Results: Findings of the timeon-task (answering speed) portion of the study were first analyzed using Levene's test for equality of variances and an independent samples t-test, which showed the experimental group to be significantly faster in answering questions one and two than the control group. Because of the smaller sample size and non-normal data, we performed a nonparametric Mann-Whitney test, which also

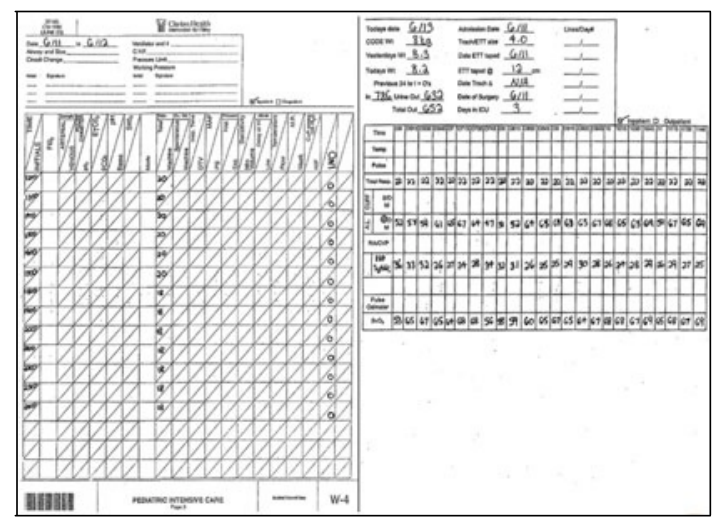

Fig. 6. Sample of traditional paper medical charts showed that tasks one and two were performed significantly faster by the experimental group than the control group. Overall, the experimental group performed six of the eight questions faster than the control group. Findings of the correct-answer data (answering accuracy) were analyzed using chi square analysis, which showed that 
participants of the experimental group performed task one correctly significantly more than participants from the control group. Because each participant from each group performed task six incorrectly, it was eliminated from the analysis. However, each task was examined on an individual basis. Therefore, the exclusion of task six did not change the results.

- Discussion: Overall, if we review all test data, for both speed and accuracy, the experimental group was generally faster and more accurate, while also being significantly faster in answering two questions. When responding to a 10 question post-task survey by the experimental group, an overall mean score of 3.78 (1-5) suggests a positive response. For example, participants agreed (with a score of 4.0) that MIVA had the potential to support critical decision-making.

\subsection{MIVA Heuristic Inspection}

As a result of these findings from the MIVA static prototype, a dynamic prototype was developed using Flash Action Script and is currently undergoing testing to ensure that it will reliably support the demanding workflow within the ICU. As part of this ongoing process, the first step has been a detailed heuristic inspection with the goal to identify and correct any common interaction and interface problems.

- Methods: In order to conduct a heuristic inspection on MIVA, we first developed a set of medical heuristics against which the device was assessed. ${ }^{1}$ The medically-relevant heuristics developed for judging the MIVA interface are shown in Table 1. The MIVA prototype was examined by three IT professionals who are also HCI graduate students in the School of Informatics, IUPUI. ${ }^{2}$

- Results and Discussion: The inspection yielded considerable data, which are consolidated in Table 2. In sum, the prototype inspection suggested that there were no catastrophic errors, but rather that the interface and interaction design of MIVA were well conceived. Only one serious issue was noted concerning possible security issues or disruptions to the data flow using the $\mathrm{HL} 7$ protocol $^{3}$ if data were to be transmitted wirelessly. This was not considered to be an interface issue, since the method of sending data to MIVA would be determined upon implementation by the health care administrator. However, a number of lower priority improvements were recommended to improve the general usability of MIVA. Some examples of these were: (1) The Minutes/Current Data and Date/Time labels must be clarified, (2) The background window of each notation should match the color of that notation's icon, (3) The background of the current-data box should be red if any of the data are out of range, and (4) A pop-up legend should be created, explaining the types of icons. Some of these changes were made immediately, and some were put on the list of items to fix during the next development iteration.

\footnotetext{
${ }^{1}$ This was accomplished by pulling together information from both software design references [12] and medical design references [13].

${ }^{2}$ We would like to acknowledge several HCI Ph.D. and MS students from the School of Informatics who helped in a portion of the discussion for this paper and their time in preparing the results of the heuristic inspections: Crystal Boston-Clay, Jay Wheeler, and Dennis Mann.

${ }^{3}$ HL7 is an international health care interoperability protocol or communication rules for the exchange, integration, sharing, and retrieval of electronic health information, while supporting clinical practice and the management, delivery, and evaluation of health services.
} 
Table 1. Medically-Relevant Heuristics

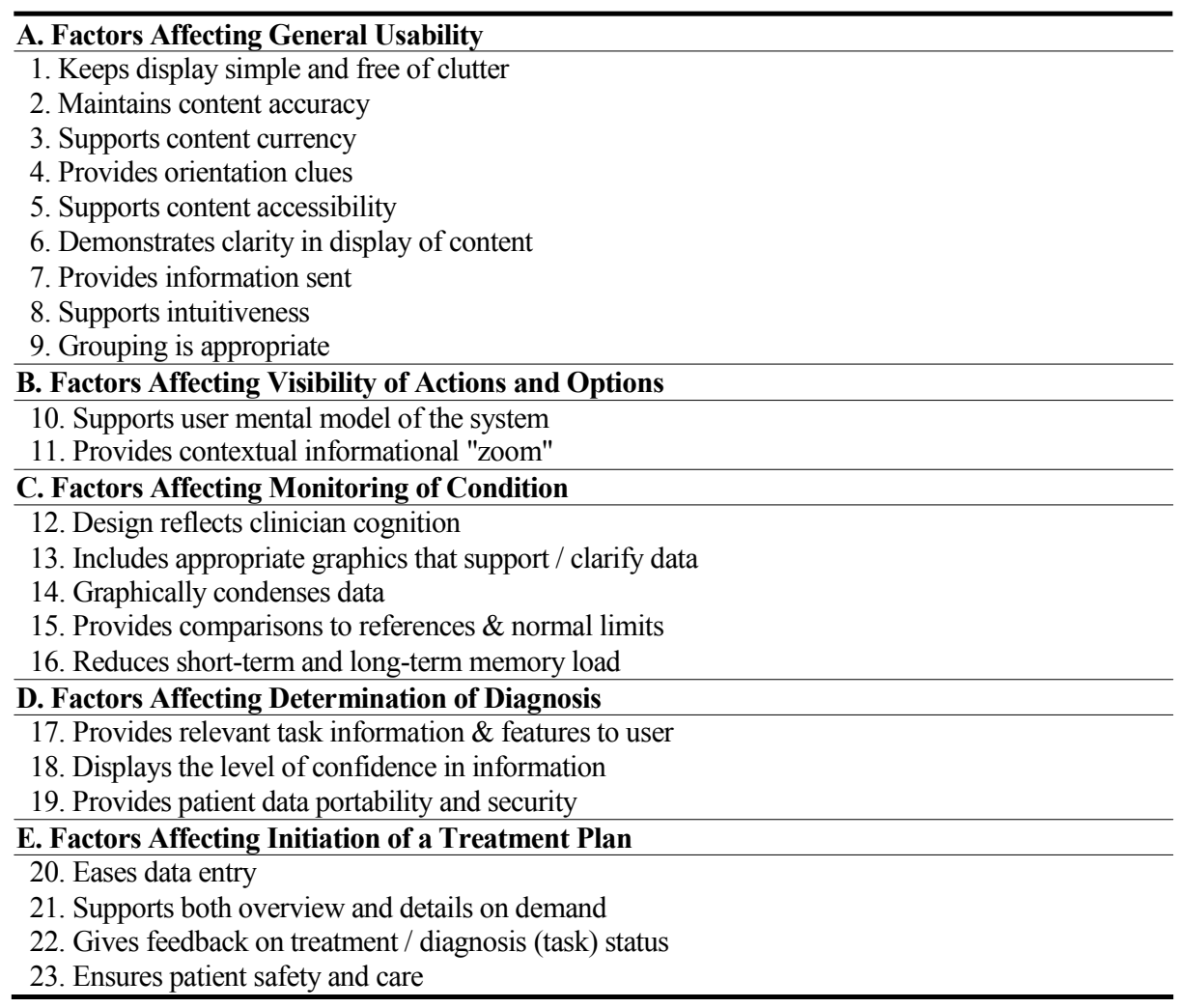

Table 2. Example of Heuristic Inspection Findings

\begin{tabular}{lll}
\hline Heuristic & Rating $\quad$ Description of Rating \\
\hline
\end{tabular}

1. Keeps display simple Medium A non-critical, limited problem (no data lost or system and free of clutter failure) that does not hinder operation and can be temporarily circumvented or avoided, but causes users moderate confusion or irritation

a. As range of elapsed time on chart grows, notations on the top of the screen become condensed and start to overlap.

b. Icons at the top of the screen illustrating notes are small and cause confusion to the user. There is not a legend provided to quickly explain each icon reference without moving the mouse over it.

c. Categorical controls that are permanently displayed should be minimized when not in use, to maximize real estate, especially for smaller form factors.

2. Maintains content Low Non-critical problems, general questions about the accuracy product, minor inconsistencies, or small aesthetic issues like labels and fields that are not aligned properly.

a. List of sets appears to have random sorting (not alphabetic); perhaps most common used?

b. The screen is consistent and users can clearly understand various sections because the labels are well-defined. 


\section{Conclusion}

Next steps for the MIVA evaluation will be a more comprehensive clinical usability study and focus group of the dynamic prototype. Methods used will replicate those used for the static prototype. In this way, comparison data will provide insights into improvements made to the interface overall. One key difference, of course, is that the data is active, rather than merely being simulated by showing multiple PowerPoint slides. This will cause dynamic and fundamental changes in the experience of the participants.

Finally, failure to follow an iterative, human-centered design methodology when creating new medical devices can impact clinical decision-making. By describing the theory behind the MIVA interface and the design and testing processed used to develop it, we hope to encourage others to use this level of care in medical product development. Both the usability study and the medically-relevant heuristic inspection can provide guidance for testing other medical and health care systems and applications.

\section{References}

1. Kohn, L.T., Corrigan, J.M., Donaldson, M.S. (eds.): To err is human: Building a safer health system. National Academies Press, Washington, DC (1999)

2. Leveson, N., Turner, C.S.: An Investigation of the Therac-25 Accidents. IEEE Computer 26(7), 18-41 (1993)

3. Bernstam, E., Hersh, W., Sim, I., Eichmann, D., Silverstein, J., Smith, J., Becich, M.: Unintended consequences of health information technology: A need for biomedical informatics. Journal of Biomedical Informatics 43(5), 828-830 (2010)

4. Zhang, J.: Human-centered computing in health information systems, Part I: Analysis and design. Journal of Biomedical Informatics 38, 1-3 (2005)

5. Horsky, J., Zhang, J., Patel, V.: To err is not entirely human: Complex technology and user cognition. Journal of Biomedical Informatics 38, 264-266 (2005)

6. Johnson, C., Johnson, T., Zhang, J.: A user-centered framework for redesigning health care interfaces. Journal of Biomedical Informatics 38, 75-87 (2005)

7. Horn, W., Popow, C., Unterasinger, L.: Support for fast comprehension of ICU data: Visualization using metaphor graphics. Methods of Information in Medicine 40, 421-424 (2001)

8. Tufte, E.R.: Visual explanations: Images and quantities, evidence and narrative. Graphic Press, Cheshire (1997)

9. Bade, R., Miksch, S., Schlechtweg, S.: Connecting time-oriented data and information to a coherent interactive visualization. In: ACM Conference on Human Factors in Computing Systems (CHI 2004), vol. 6(1), pp. 105-112 (2004)

10. Bellazzi, R., Zupan, B.: Intelligent data analysis: Special issue. Methods in Informatics Medicine 5, 362-364 (2001)

11. Faiola, A., Hillier, S.: Multivariate Relational Visualization of Complex Clinical Datasets in a Critical Care Setting: A Data Visualization Interactive Prototype. In: Proceedings of the HCI Symposium -Information Visualization Conference 2006, pp. 460-465. IEEE, London (2006)

12. Triacca, L.: MiLE+ (Milano-Lugano Evaluation method) Library of Technical Heuristics. Web Usability Enhancing Effectiveness of Methodologies and Improving their Communication Features. Ph.D. Thesis, USI COM (2005)

13. Armijo D., McDonnell C., Werner K.: Electronic Health Record Usability: Evaluation and Use Case Framework. AHRQ Publication No. 09(10)-0091-1-EF. Agency for Healthcare Research and Quality, Rockville, MD (2009) 